\title{
Cost-effectiveness of three echinocandins
}

\section{and fluconazole in the treatment of candidemia and/or invasive candidiasis in nonneutropenic adult patients}

\author{
This article was published in the following Dove Press journal: \\ ClinicoEconomics and Outcomes Research \\ 13 October 2015 \\ Number of times this article has been viewed
}

\author{
S Grau' \\ JC Pozo ${ }^{2}$ \\ E Romá ${ }^{2}$ \\ M Salavert ${ }^{3}$ \\ JA Barrueta ${ }^{4}$ \\ C Peral ${ }^{4}$ \\ I Rodriguez ${ }^{5}$ \\ D Rubio-Rodríguez ${ }^{6}$ \\ C Rubio-Terrés ${ }^{6}$ \\ 'Hospital del Mar (IMIM), Barcelona, \\ ${ }^{2}$ Hospital Universitario Reina Sofía, \\ Córdoba, ${ }^{3}$ Hospital Universitario y \\ Politécnico La Fe, Valencia, ${ }^{4}$ Pfizer \\ SLU, Alcobendas, ${ }^{5}$ Trial Form Support, \\ Madrid, ${ }^{6}$ Health Value, Madrid, Spain
}

Correspondence: D Rubio-Rodríguez Health Value, 21/5B Calle Virgen de Aránzazu, Madrid 28034, Spain

Email drubiorodriguez@healthvalue.org
Objective: To estimate the cost-effectiveness of three echinocandins (anidulafungin, caspofungin, and micafungin) and generic fluconazole in the treatment of nonneutropenic adult patients with candidemia and/or invasive candidiasis in intensive care units in Spain.

Materials and methods: A decision-tree model was applied. The success and safety (hepatic and renal adverse effects) of first-line treatments were obtained from meta-analyses and systematic reviews of clinical trials. In the case of failure, a second-line treatment (liposomal amphotericin B after the echinocandins, or one of the echinocandins after fluconazole) was administered. The duration of the treatments (14 days total) was established by a panel of clinical experts using the Delphi method and according to Infectious Diseases Society of America guidelines. The cost of the medications and renal toxicity were considered. Deterministic and probabilistic sensitivity analysis using Monte Carlo simulations were carried out.

Results: The total cost of the treatment of candidemia and/or invasive candidiasis with anidulafungin, caspofungin, micafungin, and fluconazole was $€ 5,483, € 5,968, € 6,231$, and $€ 2,088$, respectively. Anidulafungin was the dominant treatment (more effective, less expensive) compared to micafungin and caspofungin. The cost of achieving one more patient successfully treated with anidulafungin, caspofungin, and micafungin compared to fluconazole was $€ 17,199$, $€ 23,962$, and $€ 27,339$, respectively. The result remained stable, despite modification of the duration of the first-line and second-line treatments, as well as most of the dosing regimens. The probabilistic analysis also remained stable.

Conclusion: In accordance with this economic study, anidulafungin would produce savings and would be the dominant treatment compared with micafungin and caspofungin in nonneutropenic adult patients with candidemia and/or invasive candidiasis in intensive care units in Spain.

Keywords: invasive candidiasis, candidemia, anidulafungin, micafungin, caspofungin, fluconazole, cost-effectiveness analysis

\section{Introduction}

Candida spp. are the main microorganisms that cause opportunistic fungal infections in the hospital setting. Patients with increased risk for candidemia and/or invasive candidiasis (IC) are: the patients admitted to the intensive care unit (ICU), neutropenic patients with cancer, patients undergoing surgical procedures, premature infants and other immunosuppressed patients. ${ }^{1,2}$ In Spain, C. albicans is the most common species, followed by $C$. parapsilosis and C. tropicalis. ${ }^{1,3}$ The incidence of candidemia in Spain is estimated at 4.3 episodes per 100,000 inhabitants, ${ }^{1,3}$ with $33 \%-55 \%$ of episodes 
occurring in the ICU. ${ }^{1,4}$ This proportion may have declined in recent years. ${ }^{1}$ It has been estimated that the additional cost in Spain of Candida spp. infection in critically ill patients admitted to the ICU ranges approximately from $€ 8,000$ to $€ 16,000 .^{5}$

A recent guide of the European Society of Clinical Microbiology and Infectious Diseases recommends the treatment of candidemia and IC in adult patients, preferably with echinocandins (anidulafungin, micafungin, or caspofungin). ${ }^{6}$ Fluconazole is a less advisable alternative in areas with a high prevalence of non-albicans Candida, while liposomal amphotericin B presents a nonnegligible risk of renal toxicity, ${ }^{7}$ although it has shown results similar to micafungin in candidemia and IC. ${ }^{8}$

According to mixed-treatment comparison meta-analysis with fluconazole, anidulafungin is the echinocandin most likely to be the best treatment for invasive Candida infections, as opposed to micafungin, caspofungin, or liposomal amphotericin B. ${ }^{9}$

It was recently observed that critical patients with candidemia and/or IC have a high mortality rate at 30 days, greater in patients not admitted to the ICU, and that both late (30-day) and early (1-week) mortality remain high over time, being $41 \%-57 \%$ and $29 \%-39 \%$, respectively. ${ }^{10}$

The independent risk factors for mortality in the ICU at 30 days are age, the presence of an arterial line, Candida spp., preexposure to caspofungin, and the absence of antifungal treatment at the time the blood-culture results are received. ${ }^{10}$ In a recent study in Spain, the cumulative mortality at 7 and 30 days after the first episode of candidemia was $13 \%$ and $30 \%$, respectively, ${ }^{11}$ and the therapeutic measures performed within the first 48 hours (antifungal treatment and removal of vascular line) were the measures that could improve early mortality. The factors predictive of delayed death included the host characteristics (comorbidities and organ dysfunction), the primary focus of origin, and the presence of severe sepsis or septic shock.

In Spain, C. albicans is still the most common species, although others have emerged as resistant to standard treatment, such as C. parapsilosis, C. glabrata, and C. tropicalis. ${ }^{1,3}$ A study on the epidemiology of candidemia conducted in Spain in 2009 (FUNGEMYCA) revealed notable differences in the distribution of the causal species in relation to the participating hospital and ICU: C. albicans was isolated in $26 \%-72 \%$, C. parapsilosis in $11 \%-61 \%$, and C. glabrata in $3 \%-30 \%{ }^{12}$

The aim of this study was to estimate the associated cost and cost-effectiveness of three echinocandins (anidulafungin, caspofungin, and micafungin) and fluconazole (generic) in the intravenous treatment of nonneutropenic adult patients with candidemia and/or IC in ICUs in Spain, from the perspective of the National Health System.

\section{Materials and methods}

A decision-tree model was prepared in Microsoft Excel 2007 in which four options for first-line treatment (anidulafungin, micafungin, caspofungin, and fluconazole) were compared in nonneutropenic adult patients with candidemia and/or IC. Efficacy, hepatotoxicity, and nephrotoxicity results were obtained from a mixed-treatment comparison meta-analysis, ${ }^{9}$ a systematic review, ${ }^{13}$ and data from the US Food and Drug Administration, ${ }^{14}$ respectively (Figure 1). The efficacy of treatment was defined as the clinical and microbiological response to first-line treatment (anidulafungin $77.49 \%$, micafungin $75.98 \%$, caspofungin $76.10 \%$, and fluconazole $63.00 \%$ ). Hepatotoxicity was defined as the abnormal elevation to at least twofold above normal serum levels of transaminases, alkaline phosphatase, or bilirubin. Nephrotoxicity was defined as at least a doubling of the serum creatinine level, or an increase of at least $1.0 \mathrm{mg} / \mathrm{dL}$ $(88.4 \mathrm{mmol} / \mathrm{L})$ if the baseline level was elevated.

Renal toxicity does not require interrupting treatment or changing to second-line treatment. In the case of failure due to inefficacy or the occurrence of hepatic adverse effects, the administration of second-line treatment (liposomal amphotericin B after the echinocandins, or one of the echinocandins after fluconazole) was considered. This assumption in the case of liver toxicity is unfavorable to anidulafungin, because it is not necessary to interrupt treatment or switch to second-line drugs in the case of anidulafungin, in contrast with the other echinocandins. These premises, as well as the duration of first- and second-line treatments, total duration of antifungal treatment, or time horizon of the study (14 days) were established according to Infectious Diseases Society of America guidelines ${ }^{15}$ and by a panel using Delphi-type methods with clinical experts consisting of the authors of this paper: an intensive medicine specialist (JCP), infectious disease specialist (MS), and two hospital pharmacists (SG, ER). The estimated duration of first- and second-line treatment and the moment when the assessment of efficacy or hepatic or renal adverse effects should be performed are shown in Table 1.

In the economic analysis, hepatotoxicity and nephrotoxicity were chosen as the safety variables, because they are considered clinically and economically to be the most relevant antifungal toxicities. ${ }^{9,13}$ The decision tree was used 


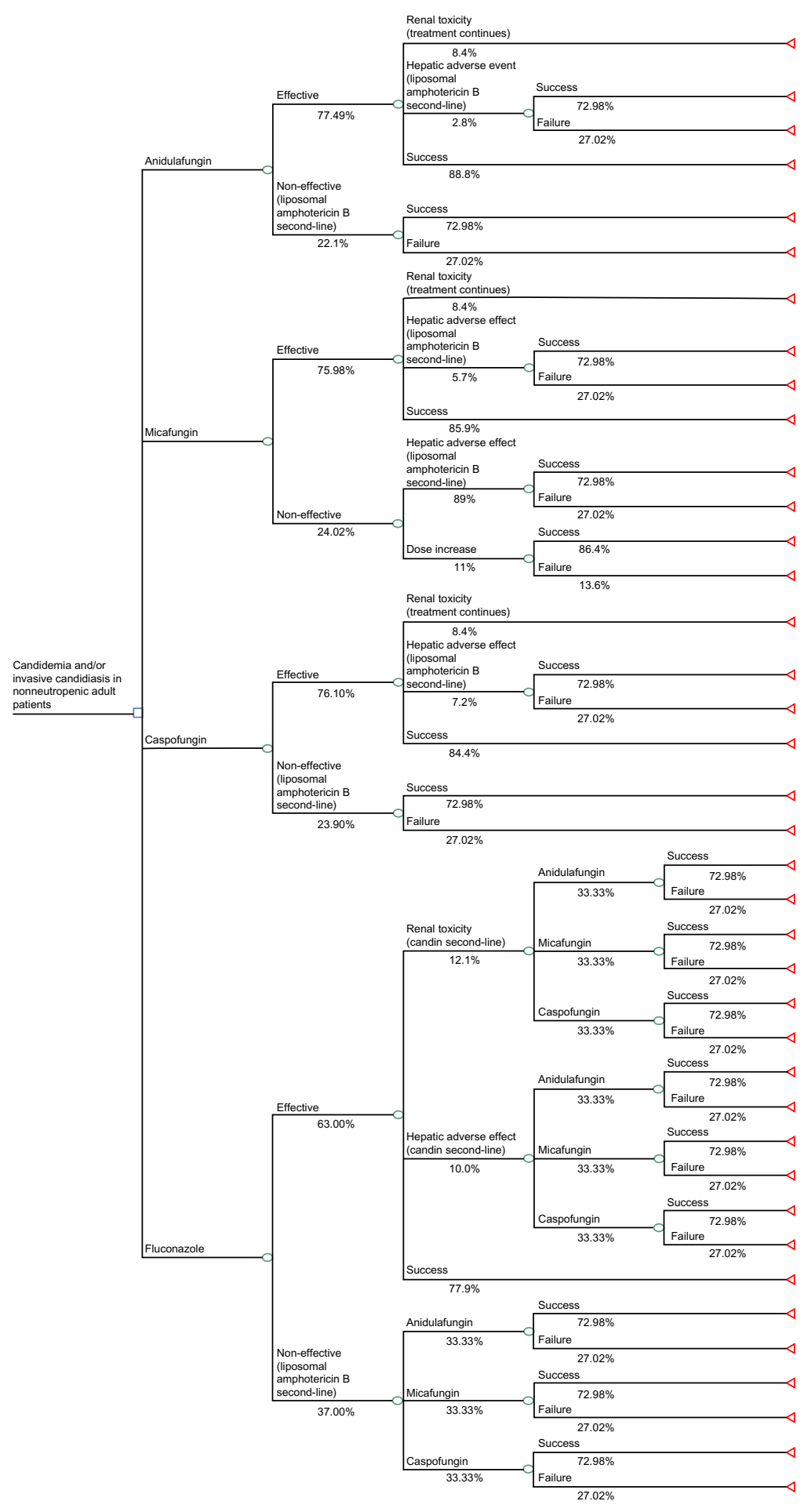

Figure I Decision-tree model.

to calculate the overall success rate of each treatment, defined as the clinical and microbiological response without hepatic adverse effects and calculated by multiplying the efficacy probability (obtained from the mixed-treatment comparison meta-analysis) ${ }^{9}$ by the likelihood of an adverse effect (hepatic or renal) ${ }^{13}$ (Table 1).
In this analysis, the cost of the medications and renal toxicity were considered, which was estimated as $€ 2,170.60$ according to a recent study by Grau et al. ${ }^{16}$ The cost of hepatotoxicity (abnormal elevation of serum transaminases, alkaline phosphatase, and bilirubin) was not considered, because it would not involve the use of additional health 
Table I Variables and assumptions of the model

\begin{tabular}{|c|c|c|}
\hline Item & Value/assumption & References/sources \\
\hline \multicolumn{3}{|l|}{ Efficacy/treatment success ${ }^{a}$} \\
\hline Anidulafungin & $77.49 \% / 68.81 \%$ & 9 (calculated) \\
\hline Micafungin & $75.98 \% / 65.27 \%$ & 9 (calculated) \\
\hline Caspofungin & $76.10 \% / 64.23 \%$ & 9 (calculated) \\
\hline Fluconazole & $63.00 \% / 49.08 \%$ & 9 (calculated) \\
\hline Liposomal amphotericin B & $72.98 \%$ & 9 \\
\hline \multicolumn{3}{|l|}{ Hepatic adverse effects ${ }^{b}$} \\
\hline Anidulafungin & $2.8 \%$ & 13 \\
\hline Micafungin & $5.7 \%$ & 13 \\
\hline Caspofungin & $7.2 \%$ & 13 \\
\hline Fluconazole & $10.0 \%$ & 13 \\
\hline \multicolumn{3}{|l|}{ Renal toxicity ${ }^{d}$} \\
\hline Anidulafungin ${ }^{c}$ & $8.4 \%(3.0 \%-11.0 \%)$ & $\mid 4,16,27$ \\
\hline Micafungin ${ }^{c}$ & $8.4 \%(3.0 \%-11.0 \%)$ & $14,16,27$ \\
\hline Caspofungin ${ }^{c}$ & $8.4 \%(3.0 \%-11.0 \%)$ & $14,16,27$ \\
\hline Fluconazole ${ }^{\mathrm{e}}$ & $12.1 \%(4.3 \%-15.8 \%)$ & 28 \\
\hline \multicolumn{3}{|l|}{ Treatment duration $^{\mathrm{d}}$} \\
\hline First-line treatment & 4 days ( $3-7$ days) & Delphi panel \\
\hline First-line effectiveness assessment & 5 th day ( $3 r d-8$ th day) & Delphi panel \\
\hline $\mathrm{AE}$ assessment/starting second-line & 5 th day ( $3 r d-8$ th day) & Delphi panel \\
\hline Second-line effectiveness assessment & 8th day ( 10 th day) & Delphi panel \\
\hline Pharmacological treatment total duration & 14 days & Delphi panel, I5 \\
\hline \multicolumn{3}{|l|}{ Drug doses (loading dose/dose increase/daily dose) } \\
\hline Anidulafungin & $200 \mathrm{mg} / \mathrm{NA} / 100 \mathrm{mg}$ & 19 \\
\hline Micafungin & $\mathrm{NA} / 200 \mathrm{mg} / \mathrm{l} 00 \mathrm{mg}$ & 20 \\
\hline Caspofungin & $70 \mathrm{mg} / \mathrm{NA} / 35$ and $50 \mathrm{mg}$ & 21 \\
\hline Fluconazole & $800 \mathrm{mg} / \mathrm{NA} / 400 \mathrm{mg}$ & 22 \\
\hline Liposomal amphotericin B & $\mathrm{NA} / \mathrm{NA} / \mathrm{I} 95 \mathrm{mg}$ & 23 \\
\hline \multicolumn{3}{|l|}{ Drug prices (ex-factory) } \\
\hline Anidulafungin (Ecalta 100 mg, one vial powder) & $€ 360.00$ & 18 \\
\hline Micafungin (Mycamine 100 mg, one vial powder) & $€ 428.57$ & 18 \\
\hline Caspofungin (Cancidas $50 \mathrm{mg}$, one perfusion vial, $10 \mathrm{~mL}$ ) & $€ 448.76$ & 18 \\
\hline Caspofungin (Cancidas $70 \mathrm{mg}$, one perfusion vial, $10 \mathrm{~mL}$ ) & $€ 570.81$ & 18 \\
\hline Fluconazole $0.2 \%$ perfusion bag, $100 \mathrm{~mL}$ & $€ 4.33$ & 18 \\
\hline Amphotericin B (Ambisome $50 \mathrm{mg}$, ten vials, $15 \mathrm{~mL}$ ) & $€ \mathrm{I}, 300.6 \mathrm{I}$ & 18 \\
\hline Amphotericin B (Abelcet $50 \mathrm{mg}$, ten vials, $10 \mathrm{~mL}$ ) & $€ 572.19$ & 18 \\
\hline
\end{tabular}

Notes: ${ }^{a}$ Effectiveness of treatment: clinical and microbiological response in the first line of treatment according to the mixed-treatment comparison meta-analysis by Mills et al. ${ }^{9}$ Treatment success: efficiency without liver or kidney adverse effects, resulting in multiplying the likelihood of effectiveness by the probability of suffering such adverse effects; ${ }^{b}$ transaminase elevation, with or without drug withdrawal; ${ }^{13}{ }^{\mathrm{c}}$ in the case of anidulafungin and micafungin, because no data were available on renal toxicity, the percentage observed with caspofungin vs fluconazole ${ }^{14,16,27}$ was assumed, assuming a class effect of echinocandins; ${ }^{d}$ values used in deterministic and probabilistic sensitivity analyses given in parentheses; eit was assumed that fluconazole renal toxicity variability was similar to that observed with echinocandins for performing the probabilistic analysis.

Abbreviations: AE, adverse effect; NA, not applicable.

resources ${ }^{17}$ other than the change of drug already foreseen in the model. The cost of the drug treatments (in 2014 euros) was calculated from the ex-factory price, ${ }^{18}$ the dosage indicated in the summary of product characteristics, ${ }^{19-23}$ and the duration of treatment estimated by the Delphi panel and according to Infectious Diseases Society of America guidelines (Table 1). The pharmacological characteristics of the echinocandins compared differ from each other and dosage changes may be required for caspofungin and micafungin. The caspofungindosing regimen depends on the patient's weight and liver function. The micafungin-dosing regimen also depends on the patient's weight. ${ }^{20,24}$ In the base case of this model, the dosing regimens of the echinocandins recommended in the summary of product characteristics for patients weighing 65 $\mathrm{kg}$ were considered. Consequently, anidulafungin is administered with a loading dose of $200 \mathrm{mg}$ followed by daily doses of $100 \mathrm{mg} .{ }^{19}$ The micafungin dose was set at $100 \mathrm{mg}$ daily for body weights of more than $40 \mathrm{~kg} .{ }^{20}$ Caspofungin is administered with a loading dose of $70 \mathrm{mg}$ followed by daily doses of $50 \mathrm{mg}$ (patients weighing less than $80 \mathrm{~kg}$ ). ${ }^{21}$ Fluconazole is administered with a loading dose of $800 \mathrm{mg}$ followed by $400 \mathrm{mg}$ daily. ${ }^{22}$ Finally, the dosing regimen of 
liposomal amphotericin B was established for a body weight of $65 \mathrm{~kg}(3 \mathrm{mg} / \mathrm{kg}){ }^{23}$

The results are presented as an incremental costeffectiveness ratio, ie, the cost of gaining an additional unit of effectiveness (overall success) with the most effective option (anidulafungin) compared with micafungin, caspofungin, and fluconazole. The least effective treatments were also compared with each other. A base case was analyzed with the mean values of all the variables; deterministic and probabilistic sensitivity analyses were made. In the deterministic analyses, the number of days of treatment and day of assessment of adverse effects occurring with first- or second-line treatment were modified (Table 1), as well as different doses and schedules for micafungin and caspofungin (according to different body weights and other factors). In the probabilistic analysis, which was carried out using the TreeAge Pro program, the effectiveness rates (overall success) of the echinocandins were obtained directly from the results of randomized clinical trials. ${ }^{8,25-28}$ A total of 1,000 Monte Carlo simulations were performed on a hypothetical cohort of 1,000 patients for the three variables (overall success rate, hepatic adverse effects, and renal toxicity rate for each treatment), which were adjusted for $\beta$-statistical distributions..$^{29}$

\section{Results}

\section{Deterministic analysis}

As shown by the base case (Table 2), anidulafungin was more effective and resulted in more therapeutic successes (68.81\%) than micafungin $(65.27 \%)$, caspofungin $(64.23 \%)$, or fluconazole $(49.08 \%)$. The total cost of treating an episode of candidemia and/or IC with these drugs was $€ 5,483$, $€ 5,968, € 6,231$, and $€ 2,088$, respectively. Anidulafungin was the dominant treatment (most effective and least expensive) compared to micafungin and caspofungin. The cost of achieving one more patient successfully treated with anidulafungin, caspofungin, and micafungin compared to fluconazole was $€ 17,199, € 23,962$, and $€ 27,339$, respectively. The resulting figures were cost-effective in every case, but particularly for anidulafungin. The results remained stable when the duration of first- and second-line treatments and dosing regimens were modified (Table 3).

\section{Probabilistic analysis}

The Monte Carlo simulation confirmed the stability of the results obtained in the base case according to $95 \%$ confidence intervals, shown in Table 4. Anidulafungin produced a saving compared to caspofungin and micafungin in $100 \%$ of the simulations (Table 4 and Figure 2). The saving per patient treated with anidulafungin ranged from $€ 449$ to $€ 487$ compared with micafungin, and from $€ 727$ to $€ 738$ compared with caspofungin. Since the estimated effectiveness of anidulafungin was greater than that of the other two echinocandins, anidulafungin was the dominant treatment (Table 4). In the comparison of anidulafungin to fluconazole, the cost of gaining an additional success with anidulafungin ranged from $€ 26,354$ to $€ 27,759$.

\section{Discussion}

In accordance with this economic study, anidulafungin produced savings and was the dominant treatment compared with micafungin and caspofungin in nonneutropenic adult patients with candidemia and/or IC in ICUs in Spain.

The prescription of echinocandins has been associated with a strong impact on budgets when only acquisition $\operatorname{costs}^{30}$ are considered. However, as this study shows, when other costs and the effectiveness of the molecules tested are considered in addition to acquisition costs, the results show that anidulafungin is the dominant alternative compared to the other echinocandins. Similarly, the additional costs of anidulafungin versus fluconazole are lower for this echinocandin compared to those derived from the use of micafungin and caspofungin. However, the efficacy of the echinocandins exceeded that of fluconazole by at least $15 \%$.

In the assessment of these results, we should take into account both the potential limitations and consistencies of the

Table 2 Base case results

\begin{tabular}{lllll}
\hline Item & Anidulafungin & Micafungin & Caspofungin & Fluconazole \\
\hline Effectiveness $^{\mathrm{a}}$ & $68.81 \%$ & $65.27 \%$ & $64.23 \%$ & $49.08 \%$ \\
Cost per branch treatment & $€ 5,483$ & $€ 5,968$ & $€ 6,231$ & $€ 2,088$ \\
$\begin{array}{l}\text { Cost per additional success } \\
\text { vs Micafungin }\end{array}$ & Dominant $^{\mathrm{b}}$ & - & & - \\
vs Caspofungin $_{\text {vs Fluconazole }}^{\mathrm{d}}$ & Dominant $^{\mathrm{b}}$ & Dominant $^{\mathrm{c}}$ & - & - \\
\hline
\end{tabular}

Notes: aEffectiveness of first-line treatment, without liver or kidney adverse effects; banidulafungin is more effective and costs less than micafungin or caspofungin; ' $m$ micafungin

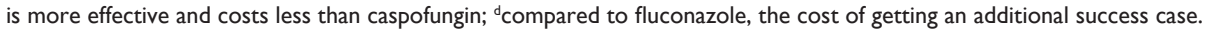




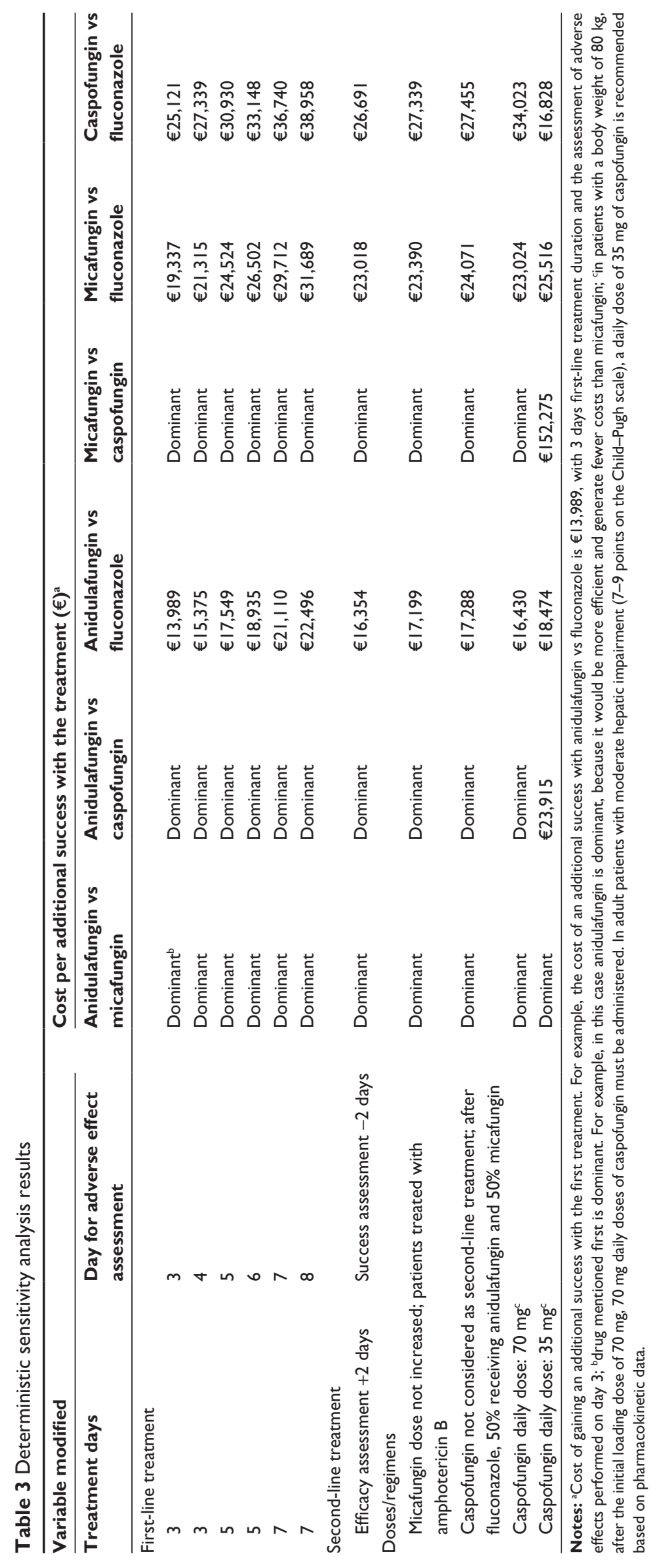


Table 4 Probabilistic analysis results (Monte Carlo simulation): anidulafungin vs comparators

\begin{tabular}{lllll}
\hline Item & Anidulafungin & Micafungin & Caspofungin & Fluconazole \\
\hline Effectiveness $^{\mathrm{a}}$ & $75.9 \% \pm 3.81 \%$ & $70.41 \% \pm 2.90 \%$ & $72.87 \% \pm 4.26 \%$ & $63.00 \% \pm 4.44 \%$ \\
Hepatic toxicity $^{\mathrm{a}}$ & $2.80 \% \pm 1.04 \%$ & $5.70 \% \pm 0.90 \%$ & $7.20 \% \pm 0.79 \%$ & $10.00 \% \pm 1.14 \%$ \\
Renal toxicity $^{\mathrm{a}}$ & $8.4 \% \pm 2.6 \%$ & $8.4 \% \pm 2.6 \%$ & $8.4 \% \pm 2.6 \%$ & $12.1 \% \pm 2.8 \%$ \\
Drug average cost (per branch) & $€ 5,494$ & $€ 5,968$ & $€ 6,226$ & $€ 2,092$ \\
$95 \% \mathrm{Cl}$ lower limit for costs & $€ 5,440$ & $€ 5,889$ & $€ 6,167$ & $€ 1,956$ \\
$95 \% \mathrm{Cl}$ upper limit for costs & $€ 5,554$ & $€ 6,04 \mathrm{I}$ & $€ 6,292$ & $€ 2,234$ \\
Cost per effectiveness unit & - & Anidulafungin is dominant & \\
\hline
\end{tabular}

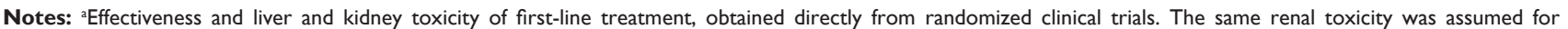
echinocandins; banidulafungin is more effective and costs less than micafungin and caspofungin.

Abbreviation: $\mathrm{Cl}$, confidence interval.

study. With regard to the limitations, it must first be remembered that this is a theoretical model, which is a simplified simulation of reality by definition. Nonetheless, costeffectiveness analysis is a particularly important instrument for facilitating the decisions of decision makers of the National Health System, because it allows integration and comparison of the effectiveness and costs of two or more options for

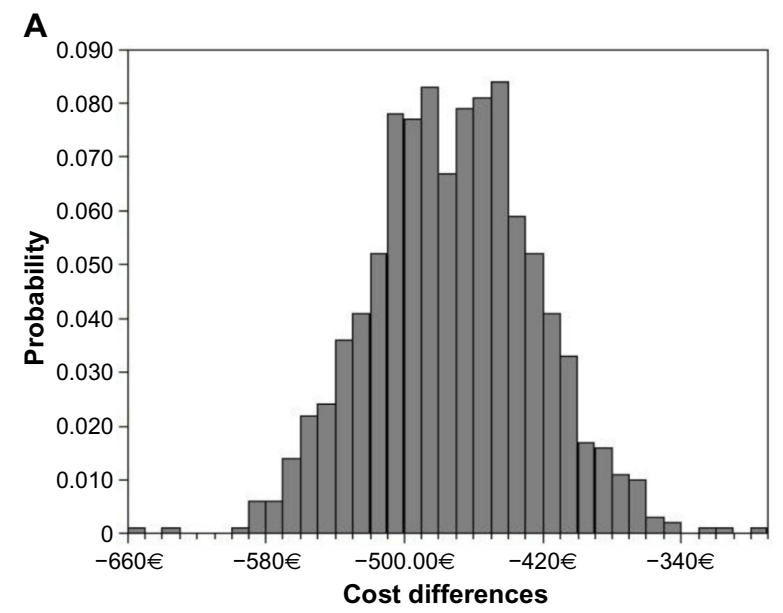

B

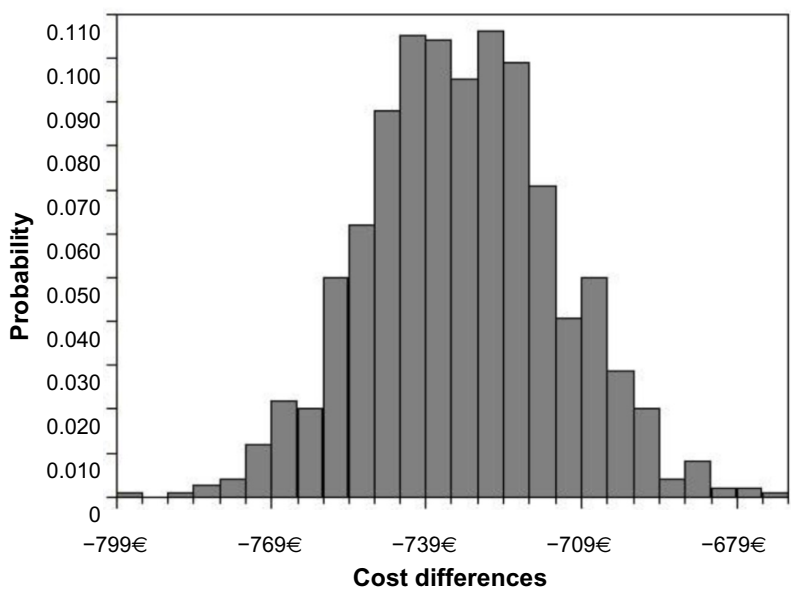

Figure 2 Probabilistic analysis (Monte Carlo simulation). Cost differences per patient.

Notes: (A) Anidulafungin vs micafungin; (B) anidulafungin vs caspofungin. an efficiency variable: in this study, the cost of gaining an additional therapeutic success with the most effective option of the drugs compared. ${ }^{31}$ Moreover, the efficacy data from which the therapeutic success rates of the drugs compared were calculated come from a mixed-treatment comparison meta-analysis ${ }^{9}$ in the deterministic analysis, and from direct clinical trial results in the case of probabilistic analysis. ${ }^{8,25-27}$ This approach (mixed-treatment comparison meta-analysis and probabilistic analysis) provides the highest possible level of evidence, according to data available.

The main consistency of the study derives from the reliability of the cost-effectiveness result obtained, as confirmed by sensitivity analysis. ${ }^{32}$ In all the deterministic sensitivity analyses, anidulafungin was the dominant treatment compared with micafungin and caspofungin. When the echinocandins were compared with fluconazole, in every scenario the cost of each additional success obtained with the echinocandins was less than $€ 30,000$, the limit considered acceptable in Spain. ${ }^{33}$ However, anidulafungin was the echinocandin that had the lowest cost per additional success versus fluconazole. Finally, according to the Monte Carlo simulation, anidulafungin versus micafungin and versus caspofungin resulted in savings in $100 \%$ of the simulations. These results corroborated the findings of the base case of the analysis.

The efficiency of treating IC with echinocandins has been evaluated in several published studies. In 2011, Wilke had published a systematic review of studies on the use and costs of anidulafungin, micafungin, and caspofungin in treating IC, concluding that the echinocandins are generally cost-effective and can even generate net savings, despite a higher acquisition cost than that of previous treatments. ${ }^{34}$ In a Spanish study published in 2012 in which the costs of treatment of IC in nonneutropenic adult patients with echinocandins were compared, it was concluded that the cost per episode and patient treated (14 days of treatment) with anidulafungin would be $€ 5,400$, the cost of caspofungin would be $€ 6,404$ in patients with a body weight of less than $80 \mathrm{~kg}$, and finally the cost 
of micafungin ranged approximately from $€ 6,000$ to $€ 9,000$, more expenses being incurred when the dose is increased due to inadequate treatment response. ${ }^{24}$ These results are similar to those obtained in this study.

Recently, in another published Spanish analysis that compared the cost-effectiveness of the treatment of candidemia with anidulafungin or fluconazole, it was concluded that the percentage of patients successfully treated with anidulafungin was higher (74\% and 57\%, respectively), at a higher drug cost (€5,991 and $€ 3,149$, respectively) but at a lower total cost ( $€ 40,047$ and $€ 41,350$, respectively), due to the reduction of other medical costs. ${ }^{16}$ This result corroborates the findings of a previous study conducted in Italy published in $2011 .{ }^{35}$ Anidulafungin was also cost-effective versus fluconazole in the treatment of candidemia, according to a study in the UK published in 2013. ${ }^{36}$ In a decision-analysis model carried out in Australia, it was found that despite the fact that the prescription of anidulafungin being associated with higher costs than fluconazole (AU\$74,587 versus AU\$ 60,945), when examining the mortality rates attributable to both drugs, anidulafungin showed that for an increase of 0.53 life-years gained, the incremental cost-effectiveness ratio would be AU\$25,740 per year of life saved, a figure considered below the threshold of costs in that country. ${ }^{37}$ Echinocandins are the recommended first-line treatment for candidemia and/or IC, given their clinical efficacy and safety. ${ }^{37}$

In accordance with this economic study, anidulafungin would produce savings and would be the dominant treatment compared with micafungin and caspofungin in nonneutropenic adult patients with candidemia and/or IC in ICUs in Spain.

\section{Acknowledgment}

This study was sponsored by Pfizer SLU, Alcobendas (Madrid), Spain.

\section{Disclosure}

The authors report no conflicts of interest in this work.

\section{References}

1. Aguado JM, Ruiz-Camps I, Muñoz P, et al. [Guidelines for the treatment of invasive candidiasis and other yeasts. Spanish Society of Infectious Diseases and Clinical Microbiology (SEIMC). 2010 Update]. Enferm Infecc Microbiol Clin. 2011;29:345-361. Spanish.

2. Pfaller MA, Diekema DJ. Epidemiology of invasive candidiasis: a persistent public health problem. Clin Microbiol Rev. 2007;20:133-163.

3. Almirante B, Rodríguez D, Park BJ, et al. Epidemiology and predictors of mortality in cases of Candida bloodstream infection: results from population-based surveillance, Barcelona, Spain, from 2002 to 2003. J Clin Microbiol. 2005;43:1829-1835.

4. Bouza E, Muñoz P. Epidemiology of candidemia in intensive care units. Int J Antimicrob Agents. 2008;32 Supp1 2:S87-S91.
5. Olaechea PM, Palomar M, León-Gil C, et al. Economic impact of Candida colonization and Candida infection in the critically ill patient. Eur J Clin Microbiol Infect Dis. 2004;23:323-330.

6. Lortholary O, Petrikkos G, Akova M, et al. ESCMID guideline for the diagnosis and management of Candida diseases 2012: patients with HIV infection or AIDS. Clin Microbiol Infect. 2012;18 Suppl 7: 68-77.

7. Kuse ER, Chetchotisakd P, da Cunha CA, et al. Micafungin versus liposomal amphotericin B for candidaemia and invasive candidosis: a phase III randomised double-blind trial. Lancet. 2007;369:1519-1527.

8. Dupont BF, Lortholary O, Ostrosky-Zeichner L, Stucker F, Yeldandi V. Treatment of candidemia and invasive candidiasis in the intensive care unit: post hoc analysis of a randomized, controlled trial comparing micafungin and liposomal amphotericin B. Crit Care. 2009;13:R159.

9. Mills EJ, Perri D, Cooper C, et al. Antifungal treatment for invasive Candida infections: a mixed treatment comparison meta-analysis. Ann Clin Microbiol Antimicrob. 2009;8:23.

10. Lortholary O, Renaudat C, Sitbon K, et al. Worrisome trends in incidence and mortality of candidemia in intensive care units (Paris area, 2002-2010). Intensive Care Med. 2014;40:1303-1312.

11. Puig-Asensio M, Padilla B, Garnacho-Montero J, et al. Epidemiology and predictive factors for early and late mortality in Candida bloodstream infections: a population-based surveillance in Spain. Clin Microbiol Infect. 2014;20:O245-O254.

12. Pemán J, Zaragoza R. [Towards an early diagnosis of invasive candidiasis in the critically ill patient]. Rev Iberoam Micol. 2012;29:71-75. Spanish.

13. Wang JL, Chang CH, Young-Xu Y, Chan KA. Systematic review and meta-analysis of the tolerability and hepatotoxicity of antifungals in empirical and definitive therapy for invasive fungal infection. Antimicrob Agents Chemother. 2010;54:2409-2419.

14. US Food and Drug Administration. Cancidas (caspofungin acetate) [prescribing information]. 2013. Available from: http://www. accessdata.fda.gov/drugsatfda_docs/label/2013/021227s0311bl.pdf. Accessed July 13, 2014.

15. Pappas PG, Kauffman CA, Andes D, et al. Clinical practice guidelines for the management of candidiasis: 2009 update by the Infectious Diseases Society of America. Clin Infect Dis. 2009;48:503-535.

16. Grau S, Salavert M, Carlos Pozo Laderas J, García Vargas M, Barrueta JA, Mir N. Cost-effectiveness of anidulafungin in confirmed candidaemia and other invasive Candida infections in Spain. J Mycol Med. 2013;23:155-163.

17. Blasco AJ, Llibre JM, Arribas JR, et al. [Analysis of costs and costeffectiveness of preferred GESIDA/National AIDS Plan regimens for initial antiretroviral therapy in human immunodeficiency virus infected adult patients in 2013]. Enferm Infecc Microbiol Clin. 2013;31:568-578. Spanish.

18. BOT Plus 2.0 [website on the Internet]. Consejo General de Colegios de Farmacéuticos. Available from: https://botplusweb.portalfarma.com. Accessed June 16, 2014.

19. Agencia Española de Medicamentos y Productos Sanitarios. Ecalta $100 \mathrm{mg}$ [summary of product characteristics]. Available from: http:// www.aemps.gob.es/cima/pdfs/es/ft/07416002/FT_07416002.pdf. Accessed June 16, 2014. Spanish.

20. Agencia Española de Medicamentos y Productos Sanitarios. Mycamine $50 \mathrm{mg}$ [summary of product characteristics]. Available from: http:// www.aemps.gob.es/cima/pdfs/es/ft/08448001/FT_08448001.pdf. Accessed June 16, 2014. Spanish.

21. Agencia Española de Medicamentos y Productos Sanitarios. Cancidas $50 \mathrm{mg}$ [summary of product characteristics]. Available from: http:// www.aemps.gob.es/cima/pdfs/es/ft/01196001/FT_01196001.pdf. Accessed June 16, 2014. Spanish.

22. Agencia Española de Medicamentos y Productos Sanitarios. Fluconazol B. Braun $2 \mathrm{mg} / \mathrm{mL}$ [summary of product characteristics]. Available from: http://www.aemps.gob.es/cima/pdfs/es/ft/71461/FT_71461.pdf. Accessed June 16, 2014. Spanish. 
23. Agencia Española de Medicamentos y Productos Sanitarios. Ambisome $50 \mathrm{mg}$ [summary of product characteristics]. Available from: http://www. aemps.gob.es/cima/pdfs/es/ft/61117/FT_61117.pdf. Accessed June 16, 2014. Spanish.

24. García-Vargas M, Casado MA, Mir N, Barrueta JA. [Cost analysis of 3 candins in the treatment of invasive candidiasis in adult nonneutropaenic patients in Spain]. Farm Hosp. 2012;36:207-215. Spanish.

25. Reboli AC, Rotstein C, Pappas PG, et al. Anidulafungin versus fluconazole for invasive candidiasis. $N$ Engl J Med. 2007;356: 2472-2482.

26. Pappas PG, Rotstein CM, Betts RF, et al. Micafungin versus caspofungin for treatment of candidemia and other forms of invasive candidiasis. Clin Infect Dis. 2007;45:883-893.

27. Mora-Duarte J, Betts R, Rotstein C, et al. Comparison of caspofungin and amphotericin B for invasive candidiasis. N Engl J Med. 2002;347: 2020-2029.

28. Kullberg BJ, Sobel JD, Ruhnke M, et al. Voriconazole versus a regimen of amphotericin $\mathrm{B}$ followed by fluconazole for candidemia in non-neutropenic patients: a randomised non-inferiority trial. Lancet. 2005;366:1435-1442.

29. Briggs A, Claxton K, Sculpher M. Decision Modelling for Health Economic Evaluation. Oxford: Oxford University Press; 2006.
30. Chen SC, Slavin MA, Sorrell TC. Echinocandin antifungal drugs in fungal infections: a comparison. Drugs. 2011;71:11-41.

31. Rubio-Terrés C, Sacristán JA, Badia X, Cobo E, García Alonso F. [Methods to perform economic assessments of health interventions]. Med Clin (Barc). 2004;122:578-583. Spanish.

32. Rubio-Terrés C, Cobo E, Sacristán JA, Prieto L, del Llano J, Badia X. [Analysis of uncertainty in the economic assessment of health interventions]. Med Clin (Barc). 2004;122:668-674. Spanish.

33. Sacristán JA, Oliva J, Del Llano J, Prieto L, Pinto JL. [What is an efficient health technology in Spain?]. Gac Sanit. 2002;16:334-343.

34. Wilke M. Treatment and prophylaxis of invasive candidiasis with anidulafungin, caspofungin and micafungin and its impact on use and costs: review of the literature. Eur J Med Res. 2011;16:180-186.

35. Reboli AC, Rotstein C, Kett DH, et al. Resource utilization and cost of treatment with anidulafungin or fluconazole for candidaemia and other forms of invasive candidiasis: focus on critically ill patients. Pharmacoeconomics. 2011;29:705-717.

36. Auzinger G, Playford G, Graham C, et al. Cost-effectiveness analysis of anidulafungin in the treatment of candidaemia. Crit Care. 2013; 17 Suppl 2:P87.

37. Neoh CF, Liew D, Slavin M, et al. Cost-effectiveness analysis of anidulafungin versus fluconazole for the treatment of invasive candidiasis J Antimicrob Chemother. 2011;66:1906-1915.
ClinicoEconomics and Outcomes Research

\section{Publish your work in this journal}

ClinicoEconomics \& Outcomes Research is an international, peerreviewed open-access journal focusing on Health Technology Assessment, Pharmacoeconomics and Outcomes Research in the areas of diagnosis, medical devices, and clinical, surgical and pharmacological intervention. The economic impact of health policy and health systems

\section{Dovepress}

organization also constitute important areas of coverage. The manuscript management system is completely online and includes a very quick and fair peer-review system, which is all easy to use. Visit http://www.dovepress.com/testimonials.php to read real quotes from published authors. 